\title{
Does Chirality Influence the Tendency toward Cocrystal Formation?
}

\author{
Fanny George, ${ }^{\dagger}$ Nikolay Tumanov, ${ }^{\dagger}$ Bernadette Norberg, ${ }^{\dagger}$ Koen Robeyns, ${ }^{\dagger}$ Yaroslav Filinchuk, ${ }^{\dagger}$ \\ Johan Wouters, ${ }^{\ddagger}$ and Tom Leyssens $*, \dagger$ \\ ${ }^{\dagger}$ Institute of Condensed Matter and Nanosciences, Université catholique de Louvain, 1348 Louvain-la-Neuve, Belgium \\ \#Unité de Chimie Physique, Théorique Et Structurale, University of Namur, Namur, Belgium
}

Supporting Information

\begin{abstract}
We performed a systematic cocrystal search for the enantiopure and racemic version of a selected active pharmaceutical ingredient, expecting that a coformer giving a cocrystal with a single enantiomer will also interact with the racemic mixture since they present identical functional groups prone to cocrystallization. We identified several novel cocrystals of levetiracetam and its racemic equivalent, etiracetam, using a wide variety of nonchiral coformers. Fourteen novel cocrystals of the enantiopure compound were obtained, whereas 18 of the

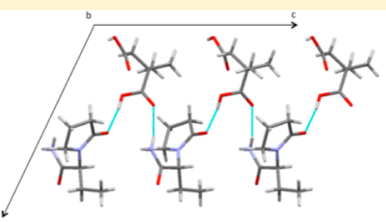

Etiracetam-

2,2-dimethylsuccinic acid cocrystal

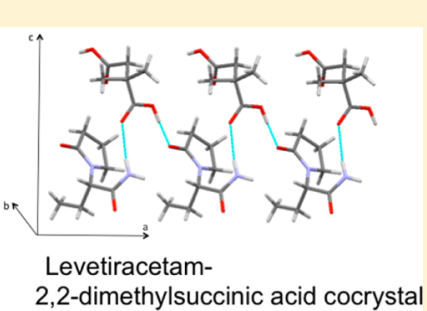

2,2-dimethylsuccinic acid cocrystal racemic compound were identified. Out of these, 13 share a common coformer. A structural analysis indicates that in most cases the strongest hydrogen bonding interactions occur both in the enantiopure, as well as the racemic cocrystal, whereas van der Waals interactions, or less strong secondary hydrogen bonding interactions, lead to a differentiation of the final structure. On the basis of our work, we suggest an approach that could lead to a more optimal cocrystal screening of an enantiopure compound, especially when a limited amount of this compound is available. Starting with a screen of the racemic compound, the set of possible coformers for the enantiopure screen can be limited to those yielding a positive hit in the former screen. Doing so, our example shows an increase in efficiency from $10 \%$ to $72 \%$.
\end{abstract}

\section{INTRODUCTION}

One of the goals of a crystallization process is to obtain a pure form, especially when working with active pharmaceutical ingredients (APIs). ${ }^{1}$ Different forms of these latter may be accessible, among them polymorphs of the pure compound, ${ }^{2,3}$ salts, ${ }^{4,5}$ hydrates, ${ }^{6,7}$ and cocrystals. ${ }^{8-10}$ Forms may differ significantly from one another in their physicochemical properties, such as solubility or bioavailability, ${ }^{11,12}$ justifying the efforts toward finding API forms with optimal properties without altering chemical identity. ${ }^{13,14}$

A multitude of drug substances are marketed as salts, but for some APIs, especially ones without ionizable groups, no viable salts can be isolated. In this case, formation of cocrystals is an interesting alternative. ${ }^{15-18}$ In the context of this work, cocrystals are considered as solids that are crystalline single phase materials composed of two or more different molecular and/or ionic compounds, called coformers, generally in a stoichiometric ratio, with components being solid under ambient conditions. They are therefore neither solvates nor salts. ${ }^{19}$

Identifying appropriate coformers able to cocrystallize with a given API may be time-consuming (trial and error process) and compound consuming, which is particularly an issue at the early stages of drug research. Consequently, attempts are made to predict matching coformers for a given compound, to improve cocrystal screening efficiency. Within this context, Springuel et al. showed that molecules presenting relatively similar chemical structures have an increased likelihood to form cocrystals with the same coformers. ${ }^{20}$ In this contribution, we use a
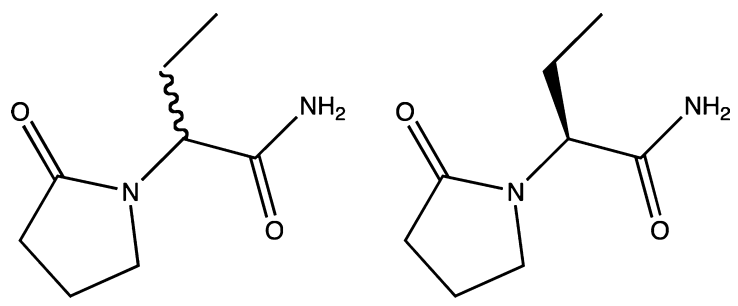

Figure 1. Chemical structures of etiracetam (left) and levetiracetam (right).

similar approach, investigating whether or not the likelihood of forming a cocrystal between an enantiopure API and a coformer is increased when this coformer was already shown to be effective toward the racemic compound. For salts, this does not seem surprising, as salt formation is mainly governed by $\mathrm{p} K_{\mathrm{a}}$ differences between base and acid. Hence an acid (or base) interacting with a given enantiopure API is also expected to interact with the racemic API. For cocrystals, this is less straightforward, as these compounds mostly depend on hydrogen-bonding interactions, which are directional in nature. In a recent contribution, ${ }^{21}$ cocrystals were shown to behave differently with respect to salts when both components are chiral; whereas for salts, formation of a diastereomeric pair

Received: February 3, 2014

Revised: April 25, 2014

Published: May 5, 2014 


\section{Table 1. Overview of Etiracetam and Levetiracetam Cocrystals}

Coform
$\begin{aligned} & \text { 1H-Pyrazole-3,5-dicarboxylic acid } \\ & \text { monohydrate }\end{aligned}$

1,3,5-benzenetricarboxylic acid

2,4-dihydroxybenzoic acid (DHBA)

3-nitrobenzoic acid (3NBA)

4-nitrobenzoic acid (4NBA)

5-nitroisophtalic acid

5-hydroxylisophthalic acid

Adipic acid

Citraconic acid

Oxalic acid (OXA)<smiles>CC(C)C(C)CC(C)C1CC(C(C)C)C(C(C)C)CC1C(C)C</smiles><smiles>CC(C)(CC(=O)O)C(=O)O</smiles><smiles>O=C(O)c1ccc(O)cc1O</smiles>

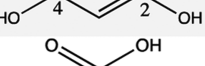<smiles>O=C(O)c1cccc(O)c1</smiles><smiles>NC(=O)c1ccc(NOC(=O)c2cccc(C(=O)O)c2)cc1</smiles><smiles>O=C(O)c1cc(O)cc(C(=O)O)c1</smiles><smiles>O=C(O)CCCCC(=O)O</smiles><smiles>C/C(=C/C(=O)O)C(=O)O</smiles><smiles>O=C(O)C(=O)O</smiles>

Cocrystals of Cocrystals of

Etiracetam Levetiracetam

$1:$

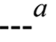

$1: 1$

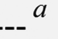

$1: 1$

$1: 1$

$: 1$

$1: 1$

$: 1$

$1: 2$

$: 2$

$1: 2$

$* b$

$1: 1$

$*^{c}$

$1: 1$ 
Table 1. continued

\begin{tabular}{|c|c|c|}
\hline Coformers & $\begin{array}{l}\text { Cocrystals of } \\
\text { Etiracetam }\end{array}$ & $\begin{array}{l}\text { Cocrystals of } \\
\text { Levetiracetam }\end{array}$ \\
\hline Oxaloacetic acid & * & $1: 1$ \\
\hline Methyl 3,4,5-trihydroxybenzoate & --- & $*$ \\
\hline 4,4-bipyridine & $*^{c}$ & $--{ }^{a}$ \\
\hline Acetoacetamide & $*$ & $*$ \\
\hline Anthranilamide & $1: 1$ & $-{ }^{c}$ \\
\hline 3-methylbutanamide & * & $*$ \\
\hline 4-chlorobenzaldéhyde & $*$ & $*$ \\
\hline Gallic acid ethyl ester & $*^{c}$ & $*^{c}$ \\
\hline
\end{tabular}

No cocrystal. ${ }^{*}$ Cocrystal identified, structure not yet determined. ${ }^{a}$ Cocrystal confirmed by slurrying experiment. ${ }^{b}$ Solvate found. ${ }^{c}$ Cocrystal confirmed by binary melting phase diagram.

seems to be the general rule, cocrystal systems are mostly enantiospecific.

To investigate how chiral APIs respond in cocrystallization toward nonchiral coformers, we performed an experimental cocrystal screen on an enantiopure and racemic version of a pharmaceutically active compound that does not form a salt. As a model compound we chose levetiracetam (LEVI), a nootropic drug used as an anticonvulsant in the treatment of epilepsy. ${ }^{22,23}$ LEVI is the biologically active enantiomeric form of (RS)-2-(2oxopyrrolidin-1-yl) butanamide, also called etiracetam (ETI) (Figure 1). Of the former, only one solid form is reported, ${ }^{24}$ while for the racemic compound two enantiotropically related polymorphs and one hydrate have been studied extensively. ${ }^{25-29}$

Previous work showed LEVI to be a potent candidate for cocrystal formation. ${ }^{20,30}$ In the current contribution, cocrystals of LEVI and ETI were identified using solvent drop grinding. $\mathrm{X}$-ray powder diffraction (XRPD) was used to detect cocrystals, while a structural comparison was carried out to compare the hydrogen-bonded network topology of enantiopure and racemic cocrystals, using crystal structures obtained from single crystal diffraction or synchrotron powder diffraction data.

\section{EXPERIMENTAL SECTION}

Starting Materials. S-2-(2-Oxopyrrolidin-1-yl)butanamide (levetiracetam, LEVI) was purchased from Xiamen Top Health Biochem Tech. Co., Ltd. 4-Nitrobenzoic acid (4NBA); adipic acid; oxalic acid (OXA); and acetoacetamide were purchased from Acros Organics. 1,3,5-Benzenetricarboxylic acid; 2,2-dimethylsuccinic acid (DMSA); 5-hydroxylisophthalic acid; oxaloacetic acid; methyl 3,4,5-trihydroxybenzoate; and 4,4-bipyridine were purchased from Alfa Aesar. 3Methylbutanamide was purchased from Maybridge. $1 \mathrm{H}$-Pyrazole-3,5dicarboxylic acid monohydrate; 2,4-dihydroxybenzoic acid (DHBA); 3-nitrobenzoic acid (3NBA); 5-nitroisophtalic acid; anthranilamide; and 4-chlorobenzaldehyde were purchased from Sigma-Aldrich. Citraconic acid and gallic acid ethyl ester were purchased from TCI. 
These materials were used as received, without further purification. (RS)-2-(2-Oxopyrrolidin-1-yl)butanamide (etiracetam, ETI) was prepared by racemization of $S$-2-(2-oxopyrrolidin-1-yl)butanamide. Ten grams of S-2-(2-oxopyrrolidin-1-yl)butanamide together with a catalytic amount (0.05 equiv) of $\mathrm{MeONa}$ was added to $10 \mathrm{~mL}$ of $\mathrm{MeOH}$. The solution was kept at reflux under continuous stirring for $24 \mathrm{~h}$ and then cooled to room temperature. The compound crystallizes spontaneously. After filtration, the compound was washed twice with $\mathrm{MeOH}$. The recovered compound was used as such.

Cocrystal Screen. Cocrystals were synthesized by solvent-drop grinding of equimolar mixtures of LEVI or ETI and a suitable coformer, with addition of $10 \mu \mathrm{L}$ of methanol. Samples were ground in a RETSCH Mixer Mill MM 400 for 90 min with a beating frequency of $30 \mathrm{~Hz}$. The resulting powders were characterized using XRPD. Comparison of the resulting diffraction pattern with the diffraction patterns of the pure phases was used to indicate cocrystal formation. All possible known forms of the pure phases were considered, to avoid confusing cocrystal formation with other phase transformations (e.g., solvate formation polymorphism).

When mixtures resulted in formation of an amorphous phase, neat (dry) grinding was performed instead. For all cocrystals identified, attempts were made to obtain a single crystal sufficiently large for structural XRD analysis.

X-ray Powder Diffraction (XRPD). X-ray diffraction measurements were performed on a Siemens D5000 diffractometer equipped with a $\mathrm{Cu}$ X-ray source operating at $40 \mathrm{kV}$ and $40 \mathrm{~mA}$ and a secondary monochromator allowing selection of the $\mathrm{K} \alpha$ radiation of $\mathrm{Cu}(\lambda=$ $1.5418 \AA$ ) . A scanning range of $2 \theta$ values from $2^{\circ}$ to $72^{\circ}$ at a scan rate of $0.6^{\circ} \mathrm{min}^{-1}$ was applied.

Synchrotron Radiation Powder X-ray Diffraction. Data for LEVI-DHBA and LEVI-DMSA cocrystals were collected at the MS-X04SA beamline at the Swiss Light Source (PSI, Switzerland) using a $1 \mathrm{D}$ microstrip detector MYTHEN II. The wavelength was $0.775045 \AA$. Wavelength and zero-shift were calibrated using a NIST 640d Si standard sample. Patterns were indexed using FOX. ${ }^{31}$ Le Bail fit was done using FOX $^{31}$ and Fullprof ${ }^{32}$ programs. Space group for each pattern was suggested taking into account analysis of systematic absences and considering that space groups have to be chiral due to presence of a chiral API molecule in the unit cell. The structure was solved by global optimization in direct space using the FOX program. ${ }^{31}$ Molecular model of LEVI, ${ }^{24}$ DMSA, ${ }^{33}$ and DHBA (using the corresponding fragment in ETI-DHBA cocrystal structure) were imported from structural models determined from single-crystal diffraction. Constraints for interatomic distances and angles were introduced: rigid group restraints were created to keep proper geometry of planar rings, amide, and carboxylic acid groups. Antibump restraints were not applied. Position, orientation, and conformation of molecules were optimized. In average, one out of 10 trials leads to a reasonable solution, so $200-300$ runs were performed to find the optimal solution. At the final stages of solution, torsion angle restraints were applied to hydrogen atoms involved in hydrogen bonding to achieve a reasonable geometry for the hydrogen bonds. The final refinement was done by the Rietveld method using the Fullprof suite, ${ }^{32}$ keeping the orientation of the molecules fixed. Unit cell parameters, coordinates of two separate molecules, scale factor, and overall temperature factor were refined. The background was described by linear interpolation between selected points determined from Le Bail fit. The final discrepancy factors are $R_{\mathrm{I}}=12.37 \%, R_{\mathrm{p}}=$ $1.33 \%, R_{\text {wp }}=1.53 \%, R_{\text {cp }}=17.03 \%$, and $R_{\text {cwp }}=18.22 \%$ for the LEVIDHBA cocrystal and $R_{\mathrm{I}}=8.75 \%, R_{\mathrm{p}}=1.25 \%, R_{\mathrm{wp}}=1.55 \%, R_{\mathrm{cp}}=$ $16.91 \%$, and $R_{\text {cwp }}=17.37 \%$ for the LEVI-DMSA cocrystal. Their refinement profiles are shown in the Supporting Information.

Using XRPD data, it is nearly impossible to differentiate cocrystals from salts. However, in the context of this work, we used a compound that does not form salts. This resolves all issues concerning the position of the hydrogen atoms.

Single Crystals. Single crystals were grown by slow evaporation from an equimolar solution of starting materials or by cooling of the corresponding supersaturated solution in suitable solvents. Different

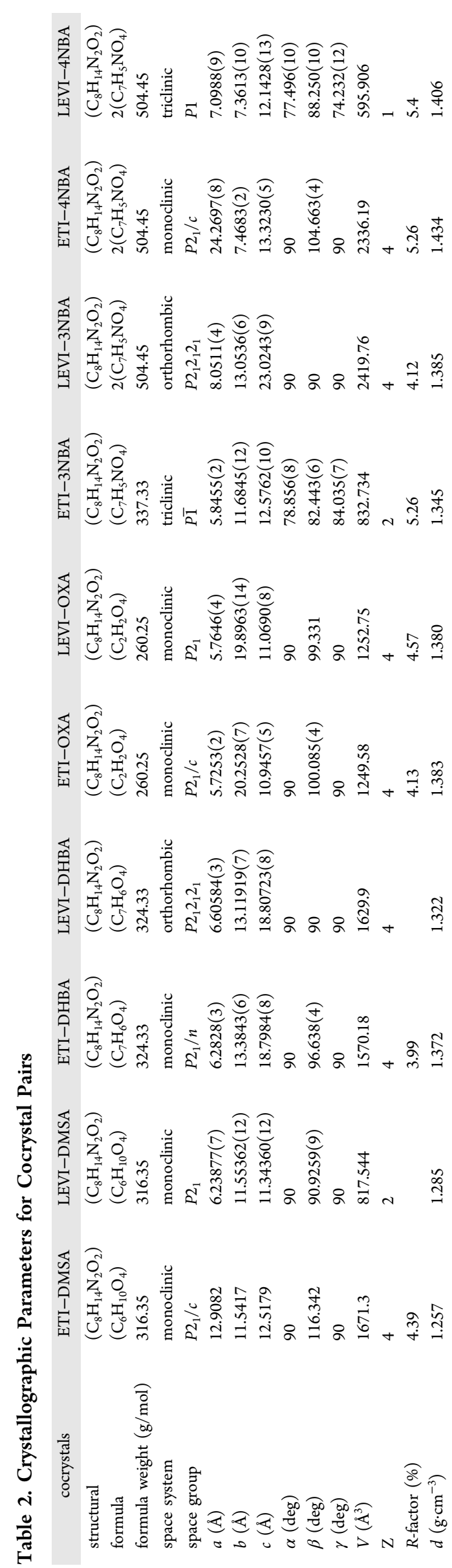




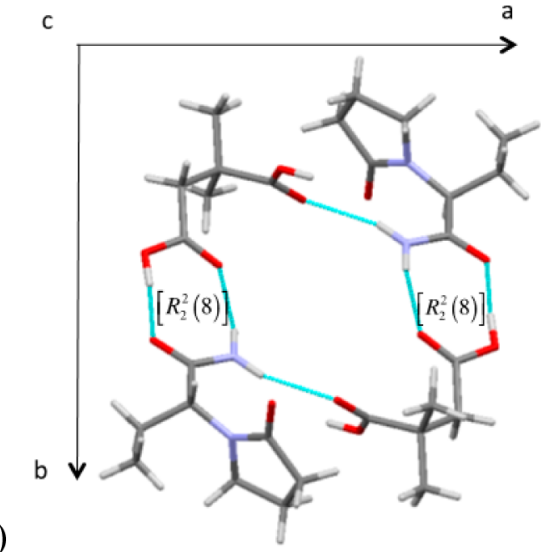

(a)

(b)

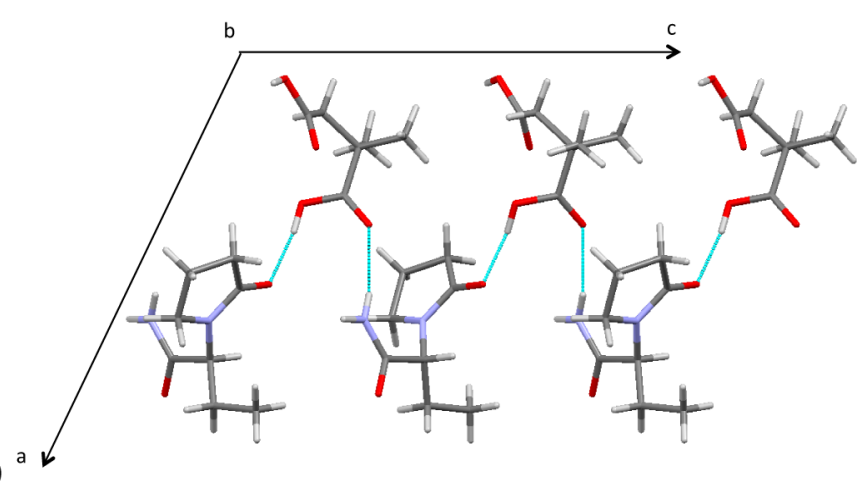

Figure 2. (a) Etiracetam and 2,2-dimethylsuccinic acid form an amide-carboxylic acid heterosynthon $\left[R_{2}^{2}(8)\right]$ ring motif and (b) a $\left[C_{2}^{2}(11)\right]$ infinite chain motif in ETI-DMSA cocrystal.

(a)

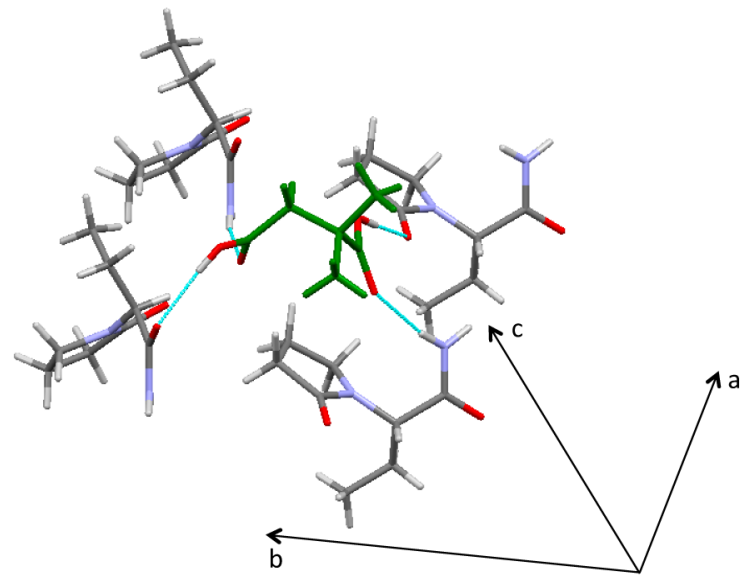

(b)

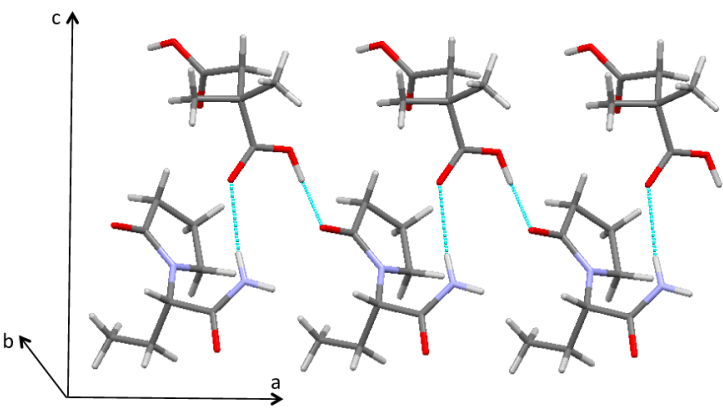

(c)

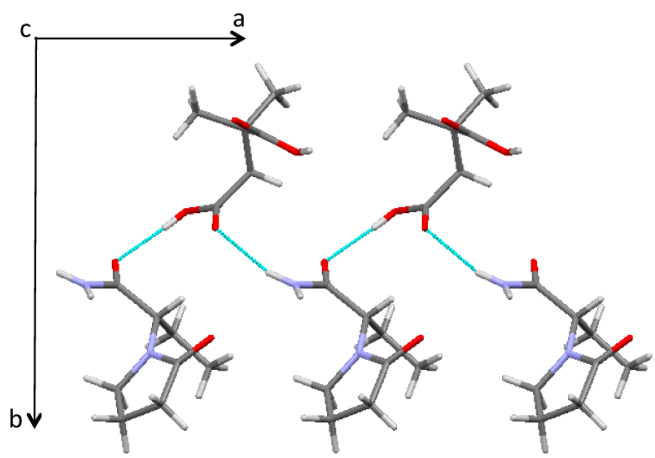

Figure 3. (a) One molecule of DMSA (green) is linked to four molecules of LEVI through four H-bonds in LEVI-DMSA cocrystal. LEVI-DMSA cocrystal shows $(\mathrm{b})$ a $\left[C_{2}^{2}(11)\right]$ infinite chain motif and $(c)$ an infinite chain with $\left[C_{2}^{2}(8)\right]$ motif.

solvents were used to increase the probability of identifying single crystals large enough for analysis.

Single Crystal X-ray Diffraction. Single crystal X-ray diffraction was performed on a Gemini Ultra R system (4-circle kappa platform, Ruby CCD detector) using $\mathrm{Cu} \mathrm{K} \alpha$ radiation $(\lambda=1.54056 \AA$ ). Cell parameters were estimated from a pre-experiment run and full data sets collected at room temperature. The structures were solved by direct methods with the SHELXS-97 program and then refined on $|F|^{2}$ using SHELXL-97 software. ${ }^{34}$ Non-hydrogen atoms were anisotropically refined, and the hydrogen atoms (not implicated in H-bonds) in the riding mode with isotropic temperature factors were fixed at 1.2 times $U(\mathrm{eq})$ of the parent atoms (1.5 times for methyl groups). Hydrogen atoms implicated in H-bonds were localized in the Fourier difference maps.

\section{RESULTS}

Hydrogen bonding is the main driving force behind cocrystal formation. As most of the typical cocrystal synthons are not affected by chirality, one could expect that a coformer yielding a cocrystal with a given chiral compound would also interact with its racemic version, as similar hydrogen bonding synthons are expected. However, in the case of cocrystals between two chiral partners, ${ }^{21}$ it was shown that the van der Waals or $\pi-\pi$ stacking 

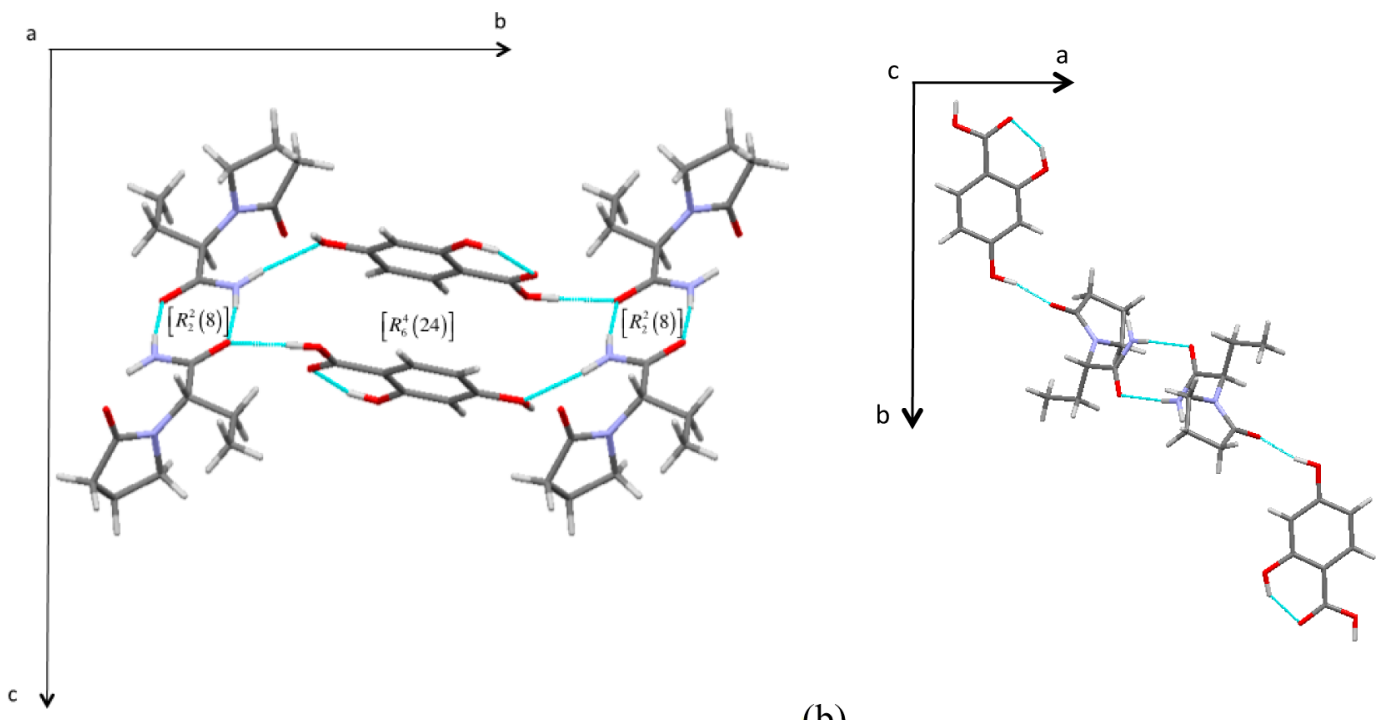

(a)

(b)

Figure 4. (a) The ETI-DHBA cocrystal shows an amide-amide homosynthon $\left[R_{2}^{2}(8)\right]$ between the noncyclic amides of two ETI and a $\left[R_{4}^{6}(24)\right]$ ring motif and (b) a dimer motif links ETI to DHBA.

interactions could be as important as the hydrogen bonding interactions and influence cocrystal stability.

To investigate the propensity toward cocrystal formation between a nonchiral partner and enantiopure or racemic compound respectively, we performed a cocrystal screen for both ETI (racemic) and LEVI (enantiopure) with a set of 152 achiral coformers. These latter were selected to have at least one alcohol, aldehyde, amide, amine, or carboxylic acid function. In total, 14 novel cocrystals of LEVI were identified, which gives a success rate for cocrystal formation of about $10 \%$. A similar number of cocrystals was also encountered for the racemic ETI compound, yielding a total of 18 novel cocrystals (Table 1). Strikingly, out of these, 13 involve a common coformer.

Although a negative test through grinding does not necessarily imply the cocrystal phase does not exist, solventdrop grinding has been shown to be an efficient method for a fast and effective detection of the majority of cocrystals. ${ }^{35}$ It is however possible that some of the coformers that were excluded in the initial screen do lead to cocrystal formation under different conditions (e.g., solvent screening, supercritical fluid crystallization, etc.). Similarly, in some cases stoichiometrically diverse cocrystals can exist. In the context of this work, we were not concerned with identifying all possible cocrystal forms between two given compounds. However, when it was evident that grinding of an equimolar mixture led to excess amount of one of the two components, different stoichiometries were tested (e.g., 1:2 mixing was done for $3 \mathrm{NBA}$ and $4 \mathrm{NBA}$ as this ratio led to full conversion according to XRPD analysis).

Out of the 32 novel cocrystals identified in this work, 15 have been structurally characterized, either through single crystal analysis or through XRPD structure determination. A pairwise comparison of enantiopure and racemic cocrystals can yield insight into the question, whether or not similar synthons are observed in both cases.

Crystal Structure Analysis. The structural analysis below focuses on the five coformers for which a cocrystal was found for both ETI and LEVI, allowing a comparison of the number and types of $\mathrm{H}$ bonding patterns formed in a pair of cocrystals. Crystallographic parameters for those 10 cocrystals are displayed in Table 2 .

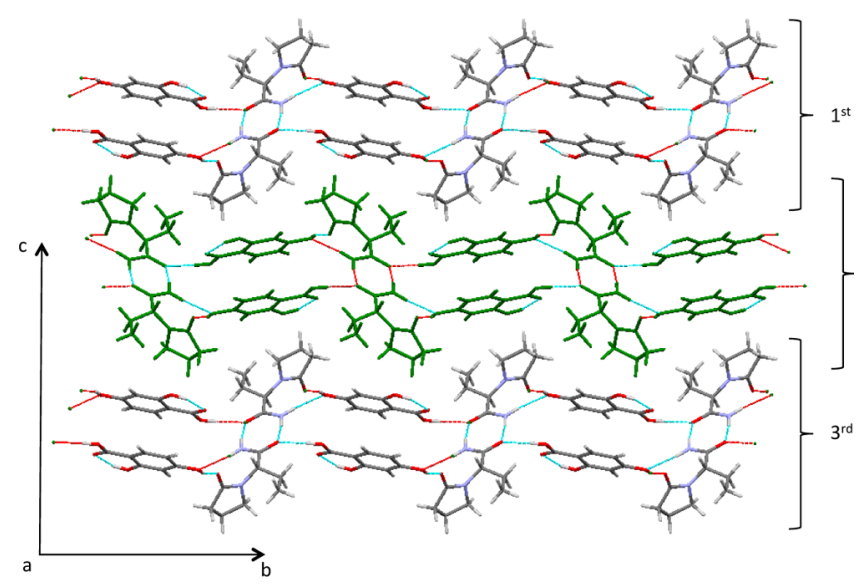

Figure 5. Stacking of three layers in ETI-DHBA cocrystal, showing infinite chain motifs $\left[C_{2}^{2}(12)\right]$ along the $c$ axis and $\pi$ stacking of molecules of DHBA acid in the $b c$ plane.

ETI-DMSA, ETI-OXA, ETI-3NBA, and ETI-4NBA cocrystals were synthesized by slow evaporation at room temperature of an equimolar, undersaturated solution of both coformers. ETI-DHBA, LEVI-OXA, LEVI-3NBA, and LEVI-4NBA were obtained by cooling crystallization to $3{ }^{\circ} \mathrm{C}$. Finally, structures of LEVI-DMSA and LEVI-DHBA cocrystals were determined from synchrotron powder diffraction data.

Etiracetam-2,2-Dimethylsuccinic Acid (1:1) (ETIDMSA). The ETI-DMSA cocrystal crystallizes from acetonitrile in the monoclinic space group $P 2_{1} / c$. The asymmetric unit contains one molecule of ETI and one molecule of DMSA.

An amide-carboxylic acid heterosynthon, described in graph set notation as $\left[R_{2}^{2}(8)\right],{ }^{36}$ is formed between the noncyclic amide of ETI and the carboxylic acid function in position 4 of DMSA (Figure 2a). In addition, the carboxylic acid function in position 1 links two molecules of ETI through a $\left[C_{2}^{2}(11)\right]$ hydrogen-bonded infinite chain motif: the acid function of DMSA acts as an acceptor of the second proton of the noncyclic amide function (the one not included in the ring motif mentioned above) and as a donor to the cyclic carbonyl function of ETI. This chain propagates along the $c$-axis (Figure $2 b$ ). 


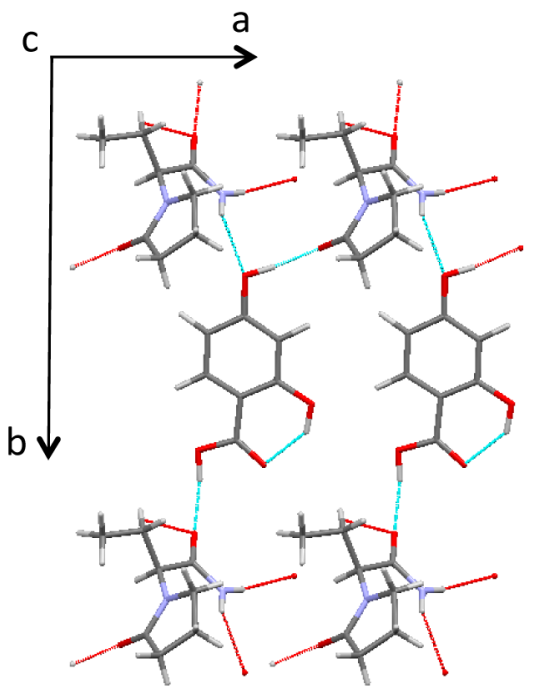

(a)

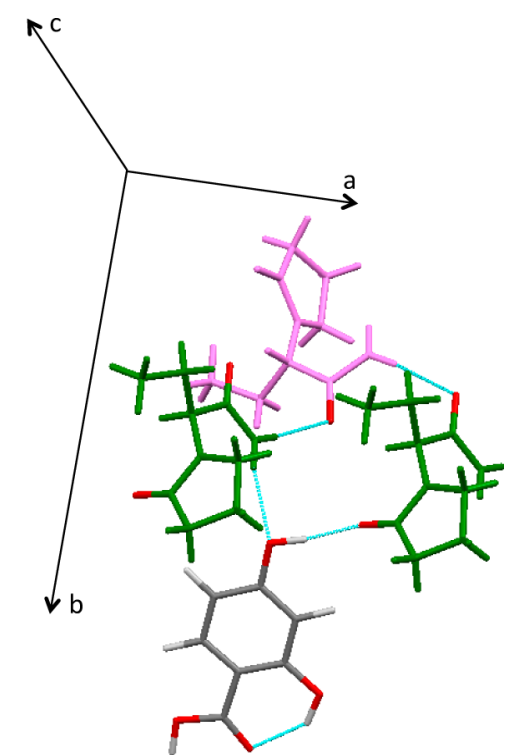

(b)

Figure 6. (a) An infinite chain motif $\left[C_{2}^{2}(12)\right]$ and a dimer motif links LEVI to DHBA acid in LEVI-DHBA cocrystal and (b) another dimer motif joins two LEVI (one pink, one green).

On the whole, the ETI-DMSA cocrystal exhibits a layered stacking, all the hydrogen bonds being located inside each layer. Secondary interactions hold the layers together due to the presence of the ethyl groups of ETI molecules and the carbonated chains of DMSA oriented outward from the layer planes.

Levetiracetam-2,2-Dimethylsuccinic acid (1:1) (LEVIDMSA). Structure of the LEVI-DMSA cocrystal was determined from synchrotron powder diffraction data in the monoclinic space group $P 2_{1}$. The asymmetric unit contains one molecule of LEVI and one molecule of DMSA.

One molecule of DMSA is linked to four molecules of LEVI through four H-bonds, two with each carboxylic acid function. The carboxylic acid in position 1 has the carbonyl group bound to a hydrogen of the noncyclic amide of a first molecule of LEVI, while the hydroxyl is connected to the cyclic carbonyl function of a second molecule of LEVI (Figure 3a), leading to a similar $\left[C_{2}^{2}(11)\right]$ hydrogen-bonding infinite chain motif as observed for the racemic ETI-DMSA cocrystal (Figure 3b). In this first cocrystal comparison, similar motifs can hence be encountered between enantiopure and racemic cocrystals. In turn the carboxylic acid group in position 4 accepts a H-bond from the noncylic amide of a third LEVI molecule while donating one to the noncyclic amide carbonyl group of a fourth LEVI molecule, creating a second infinite chain motif $\left[C_{2}^{2}(8)\right]$, along the $a$-axis (Figure 3c). In contrast with the racemic ETI-DMSA cocrystal, no amide-carboxylic acid heterosynthon $\left[R_{2}^{2}(8)\right]$ is observed, implying that not all hydrogen bonding patterns are comparable in both cases.

Overall, molecules of LEVI and DMSA are also organized in layers, which stack along the $c$ axis.

Etiracetam-2,4-Dihydroxybenzoic Acid (1:1) (ETIDHBA). The ETI-DHBA cocrystal crystallized from acetonitrile and belongs to the monoclinic space group $P 2_{1} / n$. One molecule of ETI and one molecule of DHBA occupy the asymmetric unit.

Each DHBA molecule is locked in a near planar conformation through the formation of an intramolecular $\mathrm{H}$-bond between the carboxylic acid's carbonyl group and the hydrogen of the nearest hydroxyl group (in position 2).

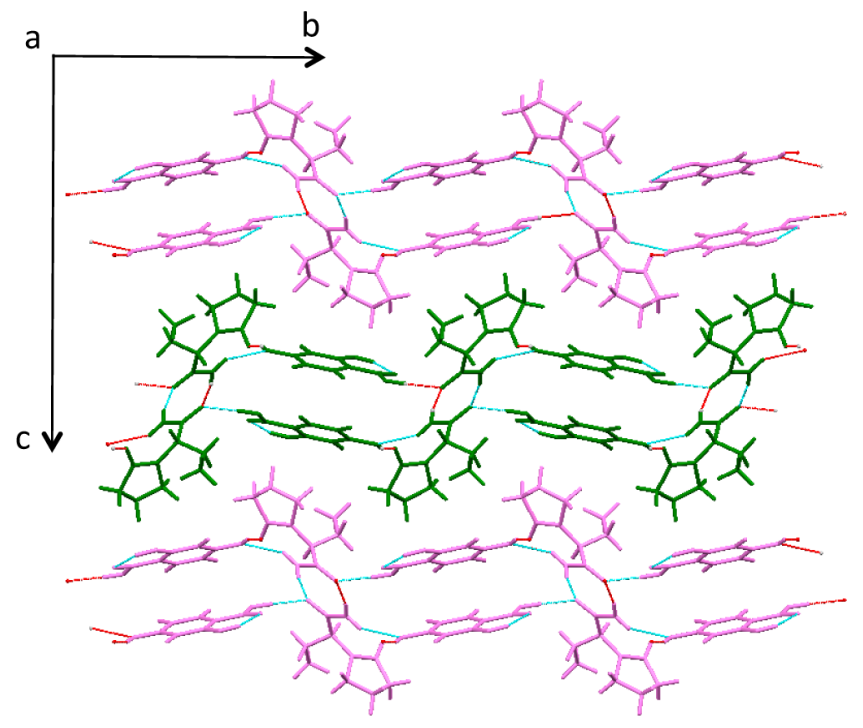

Figure 7. Stacking of three layers in LEVI-DHBA cocrystal, showing infinite chain motifs along the $b$ axis.

An amide-amide homosynthon $\left[R_{2}^{2}(8)\right]$ is formed between the noncyclic amides of two ETI molecules (Figure 4 a). A ETI...DHBA dimer motif is also observed, based on a hydrogen bond between the DHBA hydroxyl group (in position 4) and the cyclic oxygen of ETI (Figure $4 \mathrm{~b}$ ).

Here again, a $\left[C_{2}^{2}(12)\right]$ infinite chain motif is observed. This motif is formed through a first hydrogen bond donated by the DHBA carboxylic acid function to the oxygen atom of the noncyclic amide. The second hydrogen bond joins the noncyclic ETI amide with the DHBA hydroxyl group in position 4 (Figure 5).

A final structural element of interest is the $\pi$ stacking of DHBA molecules along the $c$ axis, with each successive molecule rotated by $180^{\circ} \mathrm{C}$. Consequently, two consecutive infinite chains link through a $\left[R_{6}^{4}(24)\right]$ ring motif (Figure 4a). In this motif, there are an uneven number of hydrogen acceptors and donors as the ETI oxygen atom of the noncylic amide is involved 


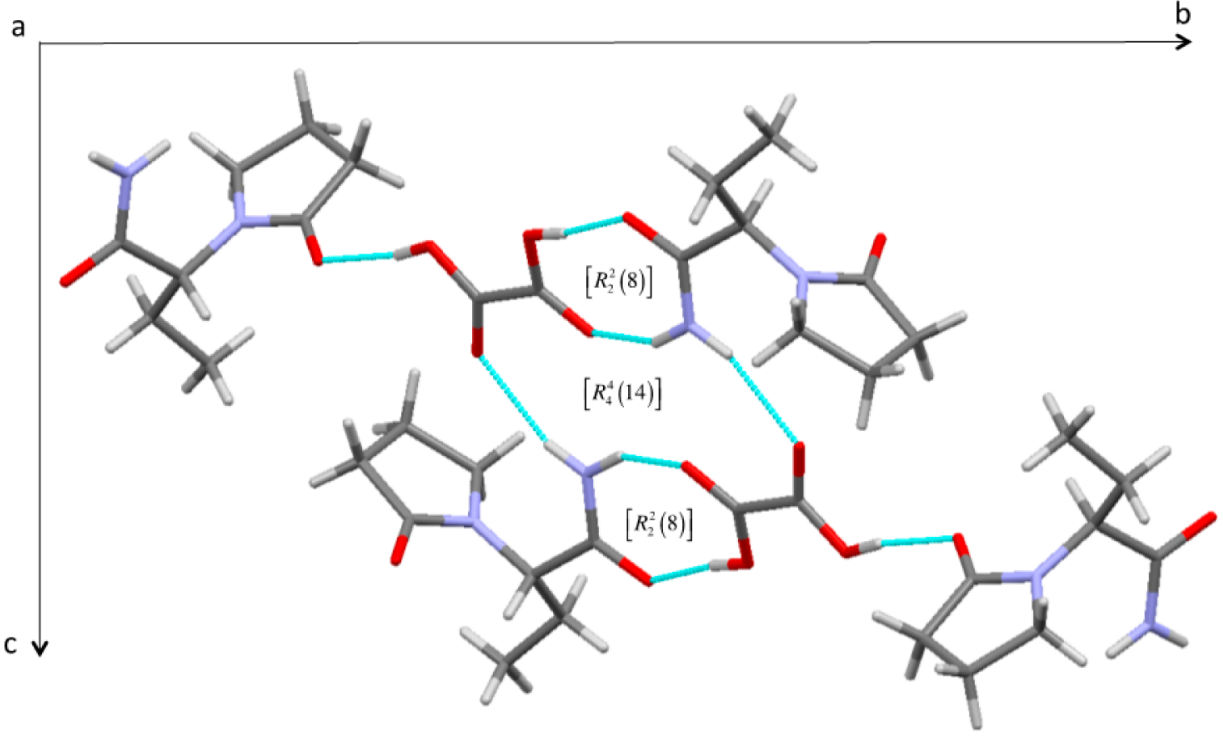

(a)

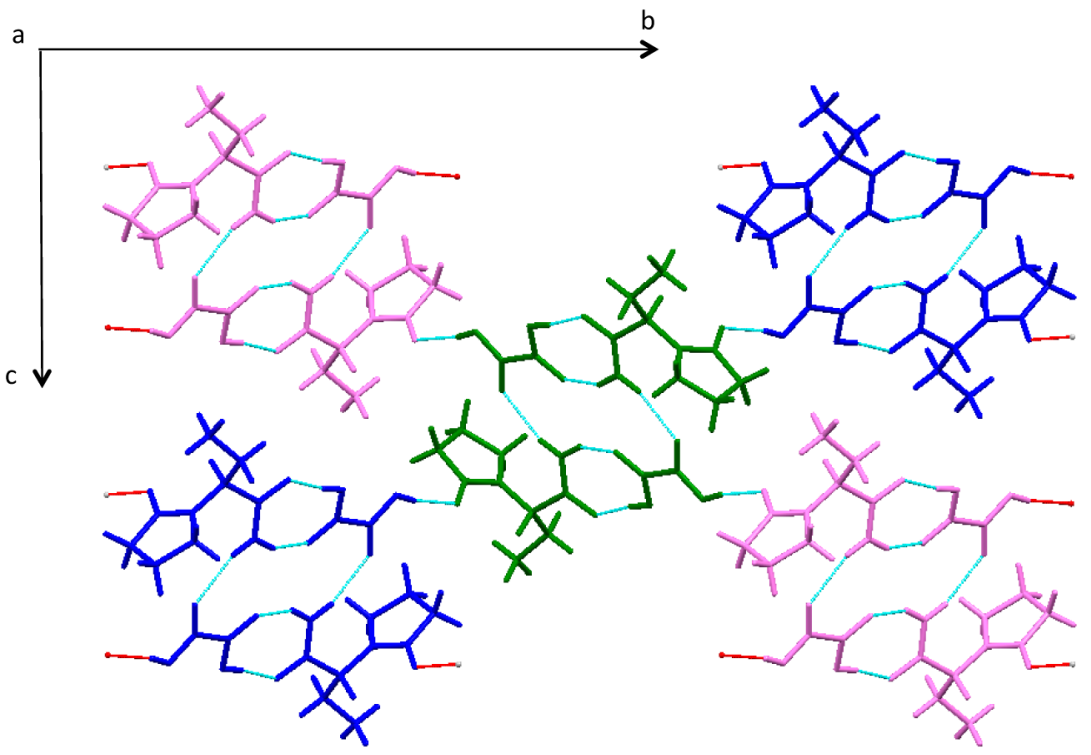

(b)

Figure 8. (a) An amide-carboxylic acid heterosynthon $\left[R_{2}^{2}(8)\right]$ is formed between ETI and OXA and a $\left[R_{4}^{4}(14)\right]$ ring motif links two ETI with two OXA, in ETI-OXA cocrystal. (b) One $\left[R_{4}^{4}(14)\right]$ ring motif (green) is linked to four identical ones (blue and pink) through hydrogen bonds.

in two different hydrogen bonds. On the whole, a close stacking of layers along the $c$ axis is observed, held together by hydrophobic interactions, due to the presence of nonpolar groups oriented outward.

Levetiracetam-2,4-Dihydroxybenzoic acid (1:1) (LEVI-DHBA). The LEVI-DHBA cocrystal was determined $a b$ initio from XRPD data and belongs to the orthorhombic space group $P 2_{1} 2_{1} 2_{1}$. The same cocrystal was crystallized from solution by Springuel et al. ${ }^{20}$ with almost identical structural parameters, confirming the validity of our results originating from synchrotron radiation data analysis. Four molecules of LEVI and four molecules of DHBA occupy the unit cell.

As in the racemic ETI-DHBA cocrystal, each DHBA molecule is locked in a near planar conformation through the formation of an intramolecular H-bond. Likewise, a similar dimer motif connects the DHBA hydroxyl group in position 4 to the LEVI cyclic amide (Figure 6a). Furthermore, the structure also shows an infinite chain motif $\left[C_{2}^{2}(12)\right]$ although constructed from slightly different hydrogen bonds. A first hydrogen bond is formed between the donating DHBA carboxylic acid function and the LEVI noncyclic amide carbonyl function, while a second hydrogen bond joins the LEVI noncyclic amide to the DHBA oxygen of the hydroxyl in position 4 (Figure 6a). This chain allows the growth of the network along the $b$ axis and shows alternating LEVI and DHBA molecules.

Although a lot of structural elements are once more similar between racemic and enantiopure cocrystals, contrary to the ETI-DHBA cocrystal, no homosynthon is found between the noncyclic amides of two LEVI molecules. Instead a supplementary hydrogen bond exists between the LEVI noncyclic carbonyl group and the proton of the noncyclic amide of a neighboring LEVI molecule (Figure 6b). As was the case for the racemic cocrystal, the carbonyl of the noncyclic amide is involved in two hydrogen bonds. 


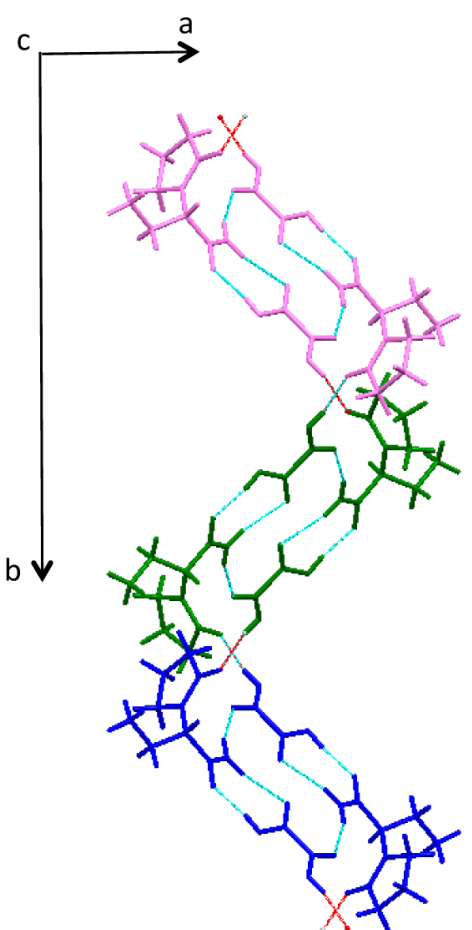

(a)

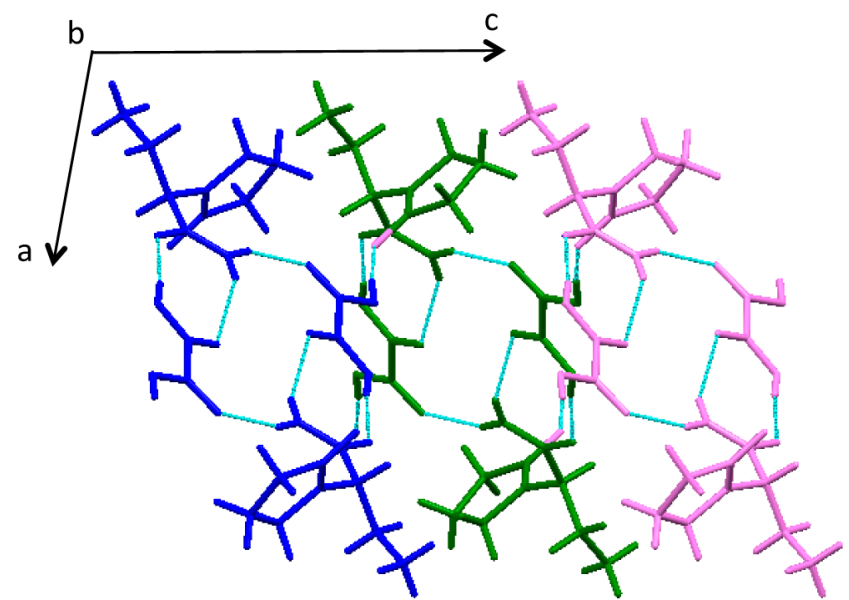

(b)

Figure 9. (a) One layer in the $a b$ plane and in (b) ac plane in ETI-OXA cocrystal.

On the whole, a close stacking of layers along the $c$ axis can be observed. In a given layer, there is no $\pi$ stacking of molecules of DHBA since those are slightly staggered along the $a$ axis. Each layer has a thickness of two molecules of DHBA or LEVI, depending on the position along the $b$ axis. Layers are held together by secondary interactions, due to the presence of the nonpolar parts of LEVI molecules oriented outward, facing the DHBA aromatic cycles. Figure 7 shows the stacking of three layers in the $b c$ plane.

Etiracetam-Oxalic Acid (1:1) (ETI-OXA). The ETIOXA cocrystal crystallizes in the monoclinic space group $P 2_{1} / c$. The asymmetric unit contains one molecule of ETI and one molecule of OXA.

An amide-carboxylic acid heterosynthon $\left[R_{2}^{2}(8)\right]$ is formed between the noncyclic amide of ETI and one of the OXA carboxylic acid functions. In addition, the second hydrogen of the ETI noncyclic amide acts as a hydrogen bond donor for the second carboxylic acid function. These two interactions link two ETI molecules with two OXA molecules forming a $\left[R_{4}^{4}(14)\right]$ ring motif (Figure 8a).

This latter ring motif binds to four similar ring motifs through a hydrogen bond between the ETI cyclic amide carbonyl group and the OXA carboxylic acid hydroxyl function not participating in the aforementioned heterosynthon (Figure $8 b$ ).

On the whole, the ETI-OXA structure is arranged in separate layers stacked along the $a$-axis. Layers are once more held together thanks to the hydrophobic groups of ETI pointing outward. Figure 9, panels a and b show one layer in the $a b$ plane and in the $a c$ plane, respectively.

Levetiracetam-Oxalic Acid (1:1) (LEVI-OXA). The enantiopure LEVI-OXA cocrystal crystallizes from acetonitrile in the monoclinic space group $P 2_{1}$. The asymmetric unit contains one molecule of LEVI and one molecule of OXA.
LEVI-OXA cocrystal parameters are almost identical to those of the ETI-OXA cocrystal. Similar hydrogen bonding motif and tridimensional patterns are observed (Figure 10).

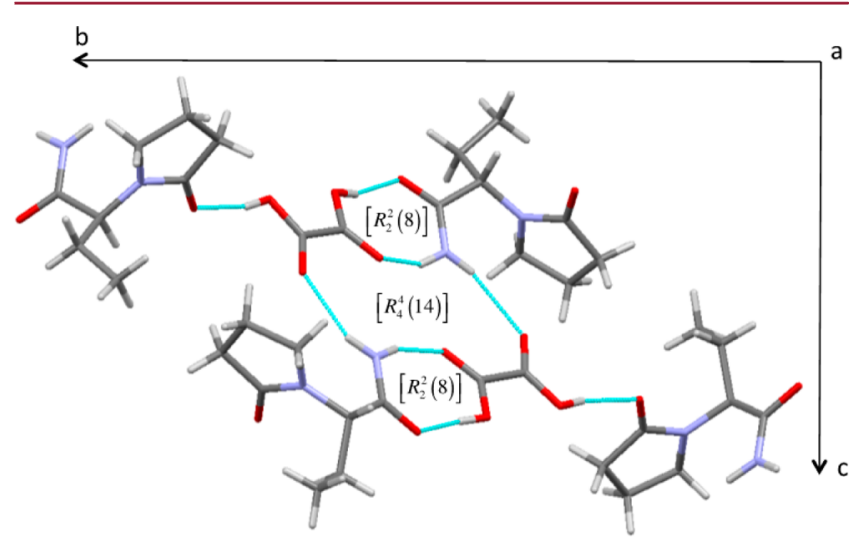

Figure 10. An amide-carboxylic acid heterosynthon $\left[R_{2}^{2}(8)\right]$ is formed between LEVI and OXA and a $\left[R_{4}^{4}(14)\right]$ ring motif links two LEVI with two OXA in LEVI-OXA cocrystal.

Etiracetam-3-Nitrobenzoic Acid (1:1) (ETI-3NBA). The ETI-3NBA cocrystal, isolated from acetonitrile, was solved in the triclinic space group $P \overline{1}$. Two molecules of ETI and two molecules of 3NBA occupy the unit cell, forming a tetramer.

In the unit cell, one observes the presence of an amidecarboxylic acid $\left[R_{2}^{2}(8)\right]$ heterosynthon between the ETI noncyclic amide and the 3NBA carboxylic acid (Figure 11a). Furthermore, two ETI molecules form an amide $\left[R_{2}^{2}(14)\right]$ homosynthon ring motif: the cyclic carbonyl of one molecule acts as an acceptor to the noncyclic $\mathrm{NH}$ of a second, resulting in the folding of the tetramer into an $S$ shape (Figure 11b). 


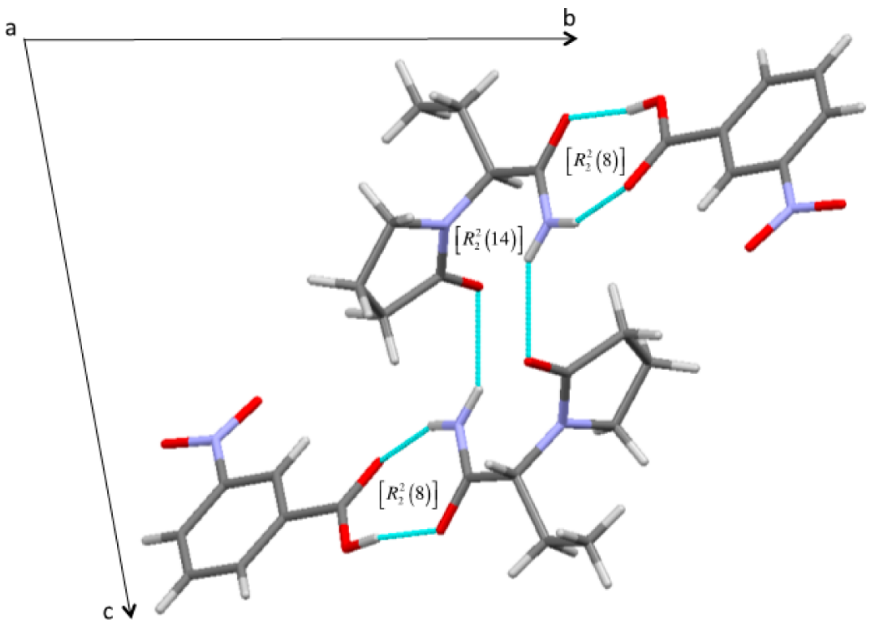

(a)

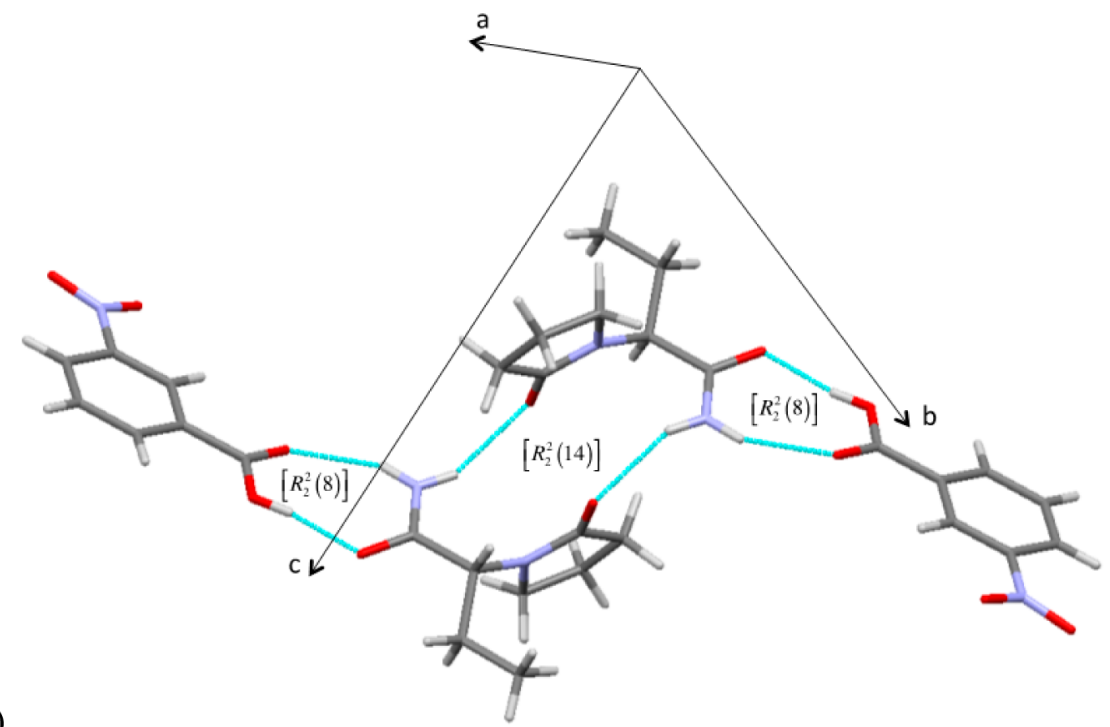

(b)

Figure 11. (a) The ETI-3NBA cocrystal exhibits an amide-carboxylic acid heterosynthon $\left[R_{2}^{2}(8)\right]$ between ETI and 3 NBA and an amide homosynthon of $\left[R_{2}^{2}(14)\right]$ ring motif between two ETI (b) An S-shape tetramer formed by two ETI and two 3NBA.

(a)

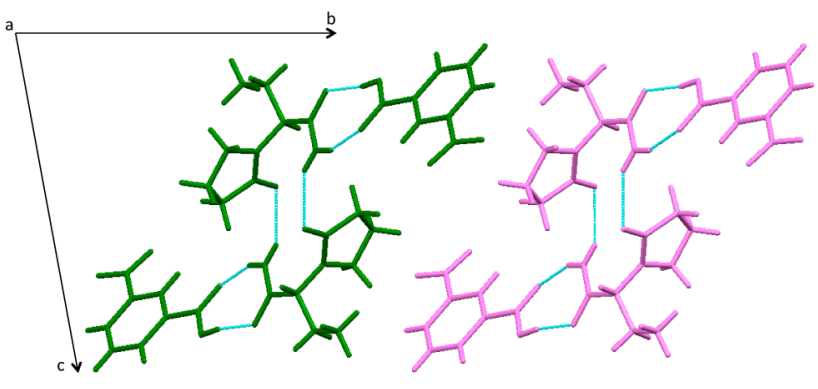

(b)

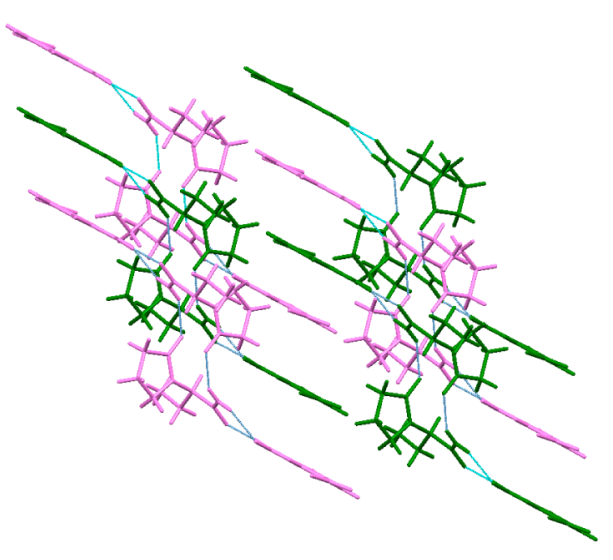

Figure 12. Stacking of tetramers (a) in the $b c$ plane and (b) in staggered rows, in ETI-3NBA cocrystal.

Tetramers are stacked in staggered rows and are linked to one another by weak hydrophobic interactions between the phenyl and pyrrolidone groups directed outward (Figure 12).

Leviteracetam-3-Nitrobenzoic Acid (1:2) (LEVI3NBA). The LEVI-3NBA cocrystal was obtained by cooling a saturated 1:2 solution (ratio suspected by XRPD) of coformers in acetonitrile and solved in the orthorhombic space group $P 2_{1} 2_{1} 2_{1}$. One molecule of LEVI and two molecules of 3NBA coexist in the asymmetric unit.

The carboxylic acid functions of these two molecules of 3NBA are involved in different hydrogen bonding motifs with one LEVI: as for the racemic version, a $\left[R_{2}^{2}(8)\right]$ heterosynthon 


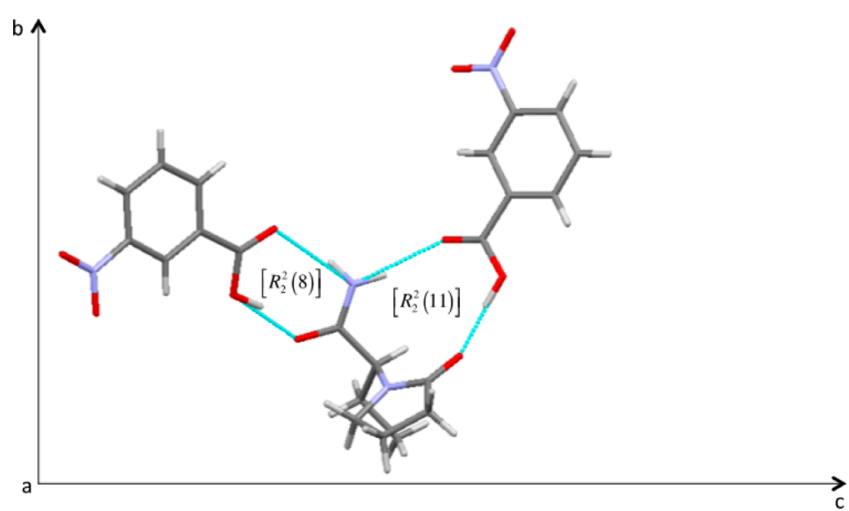

Figure 13. A $\left[R_{2}^{2}(8)\right]$ heterosynthon and a $\left[R_{2}^{2}(11)\right]$ ring motif are formed by $3 \mathrm{NBA}$ and the two LEVI molecules in the asymmetric unit cell of LEVI-3NBA cocrystal, resulting in a V-shaped trimer.

is observed for the carboxylic acid function of a first molecule, while that of the second takes part in a $\left[R_{2}^{2}(11)\right]$ ring motif (Figure 13) not observed in the enantiopure version. This motif is formed by a proton of the LEVI noncyclic amide acting as a $\mathrm{H}$ donor to the $3 \mathrm{NBA}$ carbonyl. The motif is completed by a cyclic amide carbonyl group binding to the 3NBA acid proton. These $\mathrm{H}$ bonding interactions result in the formation of a V-shaped trimer.

Overall, trimers are held together by $\pi$ stacking of 3NBA molecules and hydrophobic interactions, leading to the final 3D network.

Etiracetam-4-Nitrobenzoic Acid (1:2) (E4NBA). The ETI-4NBA cocrystal was obtained by evaporation of a 1:1 solution of coformers in acetonitrile, and solved in the monoclinic space group $P 2_{1} / c$. One molecule of ETI and two molecules of $4 \mathrm{NBA}$ coexist in the asymmetric unit.
Those two 4NBA molecules are involved in different kinds of hydrogen bonds with the ETI: the carboxylic function of the first forms a $\left[R_{2}^{2}(8)\right]$ heterosynthon with the noncylcic amide of ETI while the second takes part in a zigzag $\left[C_{2}^{2}(16)\right]$ infinite chain motif (Figure 14). In this motif, a first hydrogen bond is formed between the noncyclic amide of ETI and the nitro group of 4NBA, while a second hydrogen bond joins the ETI cyclic amide carbonyl function to the acid of 4NBA.

Overall, these bonding patterns create thin layers, densely stacked along the three directions.

Levetiracetam-4-Nitrobenzoic Acid (1:2) (LEVI4NBA). The LEVI-4NBA cocrystal was obtained by cooling a saturated 1:1 solution of coformers in ethyl acetate and solved in the triclinic space group P1. One molecule of LEVI and two molecules of 4NBA coexist in the unit cell.

The carboxylic acid functions of these two molecules of NBA are involved in different hydrogen bonding motifs with one LEVI molecule. As for the racemic version, a $\left[R_{2}^{2}(8)\right]$ acidamide heterosynthon is observed for the carboxylic acid function of a first molecule, with the LEVI noncyclic amide. The second takes part in a zigzag $\left[C_{2}^{2}(11)\right]$ infinite chain motif different from the one observed in the enantiopure version (Figure 15). In this motif, the 4NBA accepts a H-bond from the LEVI noncyclic amide and acts as a hydrogen bond donor to the LEVI cyclic carbonyl; allowing the growth of the chain along the $b$-axis. The nitro group is not involved in any hydrogen bond.

Overall, those bonding patterns form thin layers, densely stacked along the three directions, as in the ETI-4NBA cocrystal.

\section{DISCUSSION}

In this contribution, we show that enantiopure and racemic versions of a selected API, LEVI, have a tendency to form cocrystals with identical nonchiral partners. This implies that

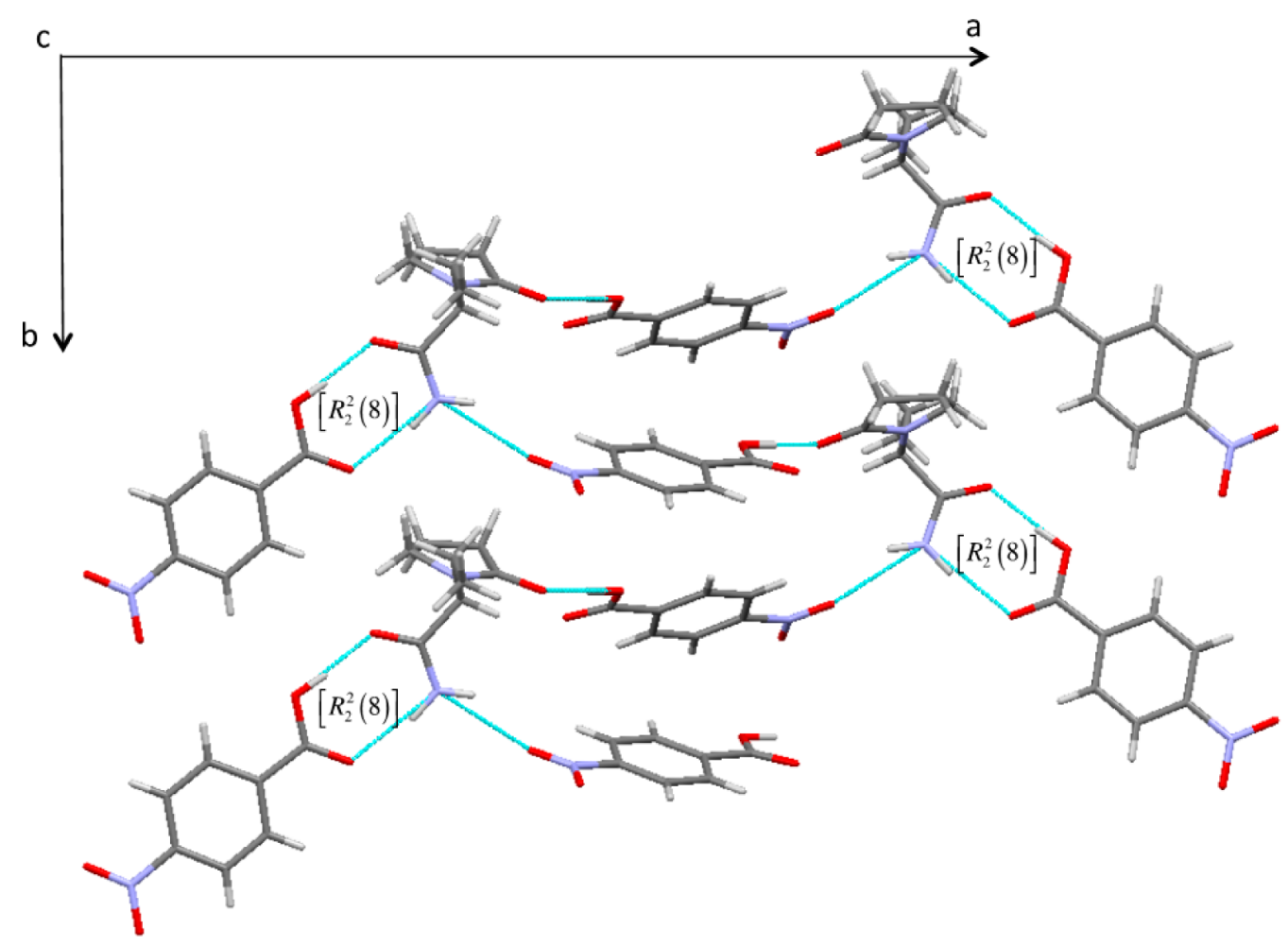

Figure 14. $\left[R_{2}^{2}(8)\right]$ acid-amide heterosynthon and the $\left[C_{2}^{2}(16)\right]$ infinite chain motif formed in the ETI-4NBA cocrystal. 


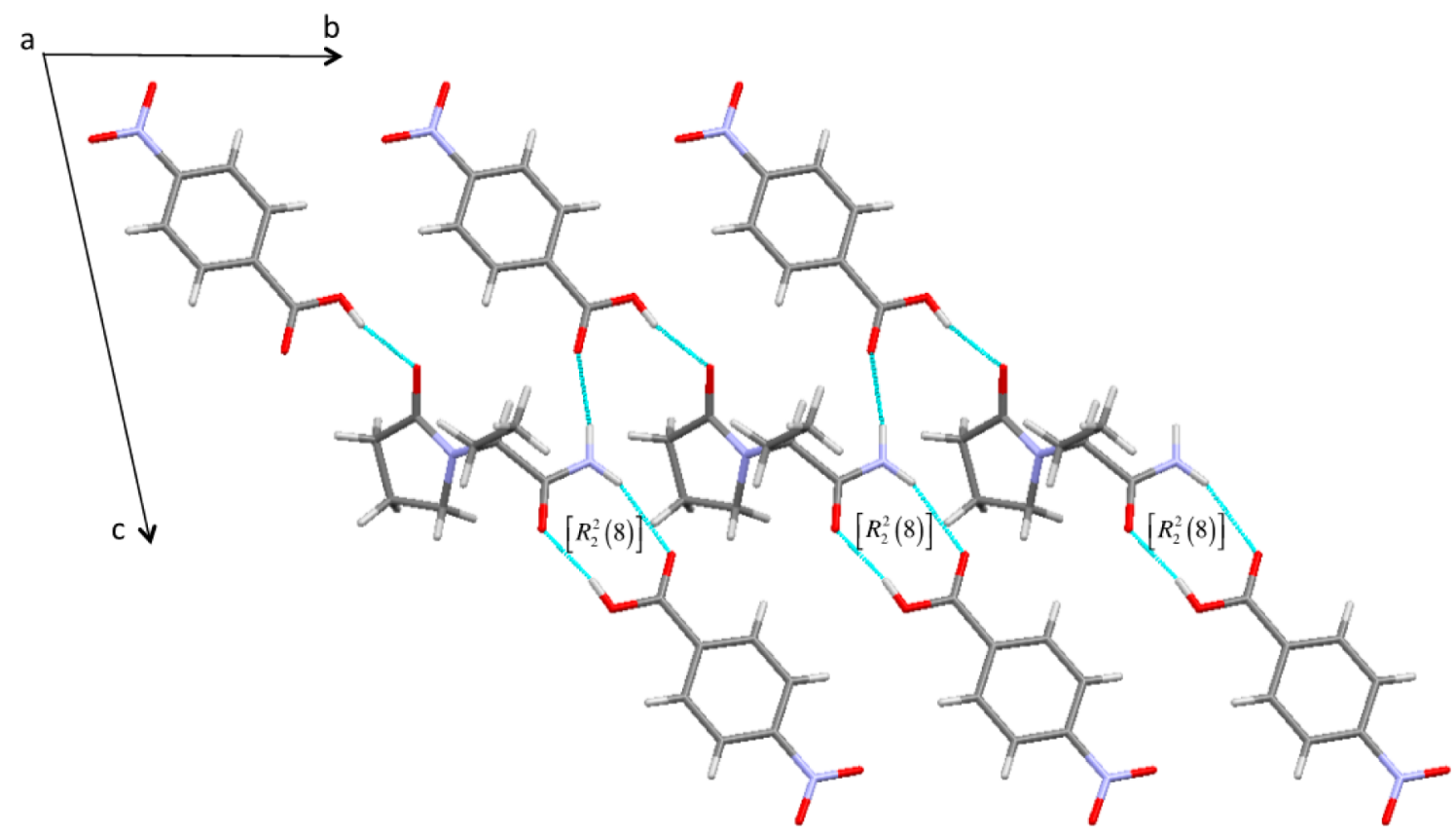

Figure 15. $\left[R_{2}^{2}(8)\right]$ acid--amide heterosynthon and the $\left[C_{2}^{2}(11)\right]$ infinite chain motif present in the LEVI-4NBA cocrystal.

cocrystal screening of an enantiopure molecule can be performed more effectively if the cocrystal screening of the racemic compound has already been performed. In early stages of drug research, the racemic compound is often more readily available, compared to the enantiopure compound. At this stage, it thus seems more interesting to perform an extended screen using the racemic compound, followed by a reduced screen with the enantiopure compound using the coformers that led to positive hits in the first screen. Our results show that if 152 coformers are tested both on the racemic as well as on the enantiopure compound, a comparable success rate of about $10 \%$ (18/152 and $14 / 152$ respectively) is observed. On the other hand, if only the reduced set of 18 coformers which gave a positive hit during the racemic screen were to be used for the enantiopure screen, a success rate of up to $72 \%$ $(13 / 18)$ is achieved. Although one out of the 14 enantiopure cocrystals would not have been identified using this approach, $90 \%$ less compound is required for the enantiopure cocrystal screen, which can be a significant advantage, as well as a speed-up for cocrystal studies in early stages of drug development.

A structural investigation into five racemic-enantiopure cocrystal pairs shows that, although not all structural elements are identical, similarities with respect to hydrogen bonding synthons can be found between the enantiopure and racemic cocrystals.

In particular the amide-acid heterosynthon is commonly encountered. In a CSD search, Steiner ${ }^{37}$ states that in $10 \%$ of cocrystal structures containing an amide and a carboxylic acid functional group, the $\left[R_{2}^{2}(8)\right]$ acid-amide heterosynthon is present. Similarly, Vishweshwar et al. ${ }^{38}$ established that this percentage increases to approximately $50 \%$ when the implied amide is a primary amide. In our structures, this element is also frequently encountered. ETI cocrystals display a $\left[R_{2}^{2}(8)\right]$ acidamide heterosynthon with all coformers but DHBA. For this latter, a $\left[R_{2}^{2}(8)\right]$ amide homosynthon is found instead. In leviteracetam cocrystals structures, these motifs are only present in LEVI-OXA, LEVI-3NBA, and LEVI-4NBA cocrystals. In the LEVI-DMSA cocrystal, the $\left[R_{2}^{2}(8)\right]$ heterosynthon is replaced by a $\left[C_{2}^{2}(8)\right]$ heterosynthon formed between one molecule of LEVI and two molecules of DMSA (or conversely).
This type of heterosynthon was already found in literature ${ }^{20}$ in the LEVI-D-tartaric acid or $S$-methylsuccinic acid cocrystals.

Our structural results show a dense hydrogen bonding network for all cocrystals. Surprisingly, each potential hydrogen donor/acceptor group of LEVI or ETI (two carbonyls and two hydrogens) is involved in hydrogen bonding patterns, contrary to the pure ETI and leviteracetam structures. This increased number of hydrogen bonds likely explains in part the propensity of this compound toward cocrystallization.

Preponderance of ETI cocrystals over LEVI stands a priori in favor of the Wallach's rule ${ }^{39}$ which states that crystals of racemic molecules show higher densities than those of enantiomeric forms, being thus more stable. ${ }^{40,41}$ This rule, however, no longer holds when the number of hydrogen bonds is important, as is the case here. Table 2 indeed shows that for some coformers, the racemic form is denser while for other coformers, the opposite is observed.

Another striking common element found in almost all cocrystals studied in this contribution (with exception of 3NBAbased cocrystals) is the formation of layered 3D networks, with layers held together by hydrophobic interactions. This shows that, although hydrogen bonding interactions are the main driving force toward cocrystal formation, possible steric hindrance and hydrophobic interactions $(\pi-\pi$ and van der Waals) also need to be taken into account when considering the overall structure. ${ }^{42-44}$

\section{CONCLUSION}

In this article, we show that enantiopure and racemic versions of a selected API tend to form cocrystals with identical nonchiral coformers. Indeed, using 152 nonchiral coformers, we identified 14 novel cocrystals of LEVI, an enantiopure compound and 18 of ETI, its racemic version. Out of these, 13 share a common coformer. A structural investigation into five racemic-enantiopure cocrystal pairs demonstrated that, although not all structural elements are identical, they often show similar hydrogen bonding synthons.

Hence, in early stages of drug research, when a racemic compound is often more readily available than its enantiopure 
counterpart, we suggest to perform an extended screen using the racemic compound, followed by a focused screen with the enantiopure one, using the coformers that led to positive hits in the first screen. In our case, this two-step approach would allow a success rate of $72 \%$ instead of $10 \%$, would require $90 \%$ less compound, and would significantly speed up cocrystal studies.

\section{ASSOCIATED CONTENT}

\section{S Supporting Information}

Structures of the racemic enantiopure cocrystal pairs described in this contribution have been deposited at the Cambridge Crystallographic Data Centre and allocated the deposition numbers 984565 for ETI-DMSA, 984566 for LEVI-DMSA, 984567 for ETI-DHBA, 984568 for LEVI-DHBA, 984569 for ETI-3NBA, 984570 for LEVI-3NBA, 984571 for ETI-4NBA, 984572 for LEVI-4NBA, 984573 for ETI-OXA, 984574 for LEVI-OXA. Additional images of these cocrystals and the refinement profiles of LEVI-DMSA and LEVI-DHBA cocrystals. This material is available free of charge via the Internet at http://pubs.acs.org.

\section{AUTHOR INFORMATION}

\section{Corresponding Author}

*Tel: +32 1047 2811. Fax: +32 104727 07. E-mail: tom. leyssens@uclouvain.be. Web: http://www.uclouvain.be/ leyssens-group.

\section{Notes}

The authors declare no competing financial interest.

\section{ACKNOWLEDGMENTS}

The authors would like to thank the UCL, UNamur and FNRS (PDR T009913F, T016913) for financial support. We acknowledge the Fonds Spéciaux de Recherche (UCL) for the incoming postdoctoral fellowship cofunded by the Marie Curie actions of the European Commission granted to N.T. The research leading to these results has received funding from the European Community's Seventh Framework Programme (FP7/2007-2013) under Grant Agreement No. 312284 (CALIPSO).

\section{REFERENCES}

(1) Billot, P.; Hosek, P.; Perrin, M.-A. Org. Process Res. Dev. 2013, 17, $505-511$.

(2) Bauer, J.; Spanton, S.; Henry, R. F.; Quick, J.; Dziki, W.; Porter, W.; Morris, J. Pharm. Res. 2001, 18, 859-866.

(3) Fabbiani, F. P. A.; Allan, D. R.; Parsons, S.; Pulham, C. R. CrystEngComm 2005, 7, 179.

(4) Kumar, V.; Malhotra, S. V. ACS Symp. Ser. 2010, 1038, 1-12.

(5) Neau, S. H. In Water-Insoluble Drug Formulation; Rong, L., Ed.; CRC Press: Boca Raton, 2000; pp 405-425.

(6) Karki, S.; Friscić, T.; Jones, W.; Motherwell, W. D. S. Mol. Pharm. 2007, 4, 347-354.

(7) Khankari, R. K.; Grant, D. J. W. Thermochim. Acta 1995, 248, 61-79.

(8) Sanphui, P.; Kumar, S. S.; Nangia, A. Cryst. Growth Des. 2012, 12, 4588-4599.

(9) Viertelhaus, M.; Hilfiker, R.; Blatter, F.; Neuburger, M. Cryst. Growth Des. 2009, 9, 2220-2228.

(10) Espinosa-Lara, J. C.; Guzman-Villanueva, D.; Arenas-García, J. I.; Herrera-Ruiz, D.; Rivera-Islas, J.; Román-Bravo, P.; Morales-Rojas, H.; Höpfl, H. Cryst. Growth Des. 2013, 13, 169-185.

(11) Cheney, M. L.; Shan, N.; Healey, E. R.; Hanna, M.; Wojtas, L.; Zaworotko, M. J.; Sava, V.; Song, S.; Sanchez-Ramos, J. R. Cryst. Growth Des. 2010, 10, 394-405.
(12) Liao, X.; Gautam, M.; Grill, A.; Zhu, H. J. J. Pharm. Sci. 2010, 99, 246-254.

(13) Rasenack, N.; Müller, B. W. Int. J. Pharm. 2002, 245, 9-24.

(14) Aaltonen, J.; Allesø, M.; Mirza, S.; Koradia, V.; Gordon, K. C.; Rantanen, J. Eur. J. Pharm. Biopharm. 2009, 71, 23-37.

(15) Aakeröy, C. B.; Grommet, A. B.; Desper, J. Pharmaceutics 2011, 3, 601-614.

(16) Thakuria, R.; Delori, A.; Jones, W.; Lipert, M. P.; Roy, L.; Rodríguez-Hornedo, N. Int. J. Pharm. 2013, 453, 101-125.

(17) Tilborg, A.; Michaux, C.; Norberg, B.; Wouters, J. Eur. J. Med. Chem. 2010, 45, 3511-3517.

(18) Pharmaceutical Salts and Co-crystals; Wouters, J.; Quéré, L., Eds.; Royal Society of Chemistry: Cambridge, UK, 2011.

(19) Aitipamula, S.; Banerjee, R.; Bansal, A. K.; Biradha, K.; Cheney, M. L.; Choudhury, A. R.; Desiraju, G. R.; Dikundwar, A. G.; Dubey, R.; Duggirala, N.; Ghogale, P. P.; Ghosh, S.; Goswami, P. K.; Goud, N. R; Jetti, R. R. K. R.; Karpinski, P.; Kaushik, P.; Kumar, D.; Kumar, V.; Moulton, B.; Mukherjee, A.; Mukherjee, G.; Myerson, A. S.; Puri, V.; Ramanan, A.; Rajamannar, T.; Reddy, C. M.; Rodriguez-Hornedo, N.; Rogers, R. D.; Row, T. N. G.; Sanphui, P.; Shan, N.; Shete, G.; Singh, A.; Sun, C. C.; Swift, J. A.; Thaimattam, R.; Thakur, T. S.; Kumar Thaper, R.; Thomas, S. P.; Tothadi, S.; Vangala, V. R.; Variankaval, N.; Vishweshwar, P.; Weyna, D. R.; Zaworotko, M. J. Cryst. Growth Des. 2012, 12, 2147-2152.

(20) Springuel, G.; Norberg, B.; Robeyns, K.; Wouters, J.; Leyssens, T. Cryst. Growth Des. 2012, 12, 475-484.

(21) Springuel, G.; Robeyns, K.; Norberg, B.; Wouters, J.; Leyssens, T. Cryst. Growth Des. 2014, submitted.

(22) Sekhon, B. S. ARS Pharm. 2009, 50, 99-117.

(23) Hurtado, B.; Koepp, M. J.; Sander, J. W.; Thompson, P. J. Epilepsy Behav. 2006, 8, 588-592.

(24) Herman, C.; Vermylen, V.; Norberg, B.; Wouters, J.; Leyssens, T. Acta Crystallogr. B. Struct. Sci. Cryst. Eng. Mater. 2013, 69, 371-378.

(25) Herman, C.; Haut, B.; Aerts, L.; Leyssens, T. Int. J. Pharm. 2012, $437,156-161$.

(26) Herman, C.; Haut, B.; Douieb, S.; Larcy, A.; Vermylen, V.; Leyssens, T. Org. Process Res. Dev. 2012, 16, 49-56.

(27) Herman, C.; Leyssens, T.; Debaste, F.; Haut, B. J. Cryst. Growth 2012, 342, 57-64.

(28) Herman, C.; Haut, B.; Halloin, V.; Vermylen, V.; Leyssens, T. Org. Process Res. Dev. 2011, 15, 774-782.

(29) Herman, C.; Leyssens, T.; Vermylen, V.; Halloin, V.; Haut, B. J. Chem. Thermodyn. 2011, 43.

(30) Springuel, G.; Leyssens, T. Cryst. Growth Des. 2012, 12, 33743378.

(31) Favre-Nicolin, V.; Černý, R. J. Appl. Crystallogr. 2002, 35, 734743.

(32) Rodríguez-Carvajal, J. Phys. B Condens. Matter 1993, 192, 5569.

(33) Özcan, Y.; Osmanoglu, S.; Ide, S. Anal. Sci. 2003, 19, 12211222.

(34) Sheldrick, G. M. Acta Crystallogr., Sect. A 2008, 64, 112-122.

(35) Delori, A.; Friščić, T.; Jones, W. CrystEngComm 2012, 14, 2350.

(36) Etter, M. C.; MacDonald, J. C.; Bernstein, J. Acta Crystallogr., Sect. B 1990, 46, 256-262.

(37) Steiner, T. Angew. Chemie Int. Ed. 2002, 41, 48-76.

(38) Vishweshwar, P.; McMahon, J. a; Bis, J. a; Zaworotko, M. J. J. Pharm. Sci. 2006, 95, 499-516.

(39) Wallach, O. Justus Liebig's Ann. Chem. 1895, 286, 90-118.

(40) Friscić, T.; Fábián, L.; Burley, J. C.; Reid, D. G.; Duer, M. J.; Jones, W. Chem. Commun. (Cambridge) 2008, 1644-1646.

(41) Brock, C. P.; Schweizer, W. B.; Dunitz, J. D. J. Am. Chem. Soc. 1991, 113, 9811-9820.

(42) Baures, P. W.; Rush, J. R.; Wiznycia, A. V.; Desper, J.; Helfrich, B. A.; Beatty, A. M. Cryst. Growth Des. 2002, 2, 653-664.

(43) Takata, N.; Shiraki, K.; Takano, R.; Hayashi, Y.; Terada, K. Cryst. Growth Des. 2008, 8, 3032-3037.

(44) Vishweshwar, P.; Nangia, A.; Lynch, V. M. Cryst. Growth Des. 2003, 3, 783-790. 